\title{
Wissenschaftliche Monographien im digitalen Zeitalter: Die neue Open-Access-Politik des Schweizerischen Nationalfonds
}

\author{
Daniel Krämer"
}

\section{A. Einleitung}

Die Empörung war gross, als der Schweizerische Nationalfonds (SNF) im Frühling 2014 wissenschaftliche Monographien in seine Open-Access-Politik einschloss. Kritische Stimmen monierten, mit dem Zwang zu einer Open-Access-Veröffentlichung schaffe der SNF ein "digitales Ghetto", er verhindere Forschung, statt sie zu fördern und durch seinen vorschnellen und unilateralen Entscheid wolle er auf Kosten der Sozial- und Geisteswissenschaften sparen. ${ }^{1}$ Sie bemängelten zudem, der SNF trage den unterschiedlichen Wissenschafts- und Publikationskulturen nicht genügend Rechnung, zumal Monographien auch in Bibliotheken für die Öffentlichkeit kostenlos zugänglich seien. ${ }^{2}$ Wohlwollende Stimmen teilten nicht nur das Ziel des SNF, Forschungsergebnisse im digitalen Zeitaltern weltweit und schnell zu verbreiten, sondern wiesen auch auf bestehende Zugangsbarrieren hin, bezweifelten den Nutzen eines Druckzwangs bei Dissertationen und machten allgemein auf eine zunehmende Zahl von Neuerscheinungen bei sinkenden Verkaufszahlen aufmerksam («monograph crisis»). ${ }^{3}$

Zum ersten Mal wurde Open Access in der Schweiz in den Medien und in einer breiteren Öffentlichkeit diskutiert. ${ }^{4}$ Knapp 4200 Personen unterzeichneten innert weniger Wochen einen Onlineaufruf zur Rettung der akademischen Verlage in der Schweiz. ${ }^{5}$ Rechtswissenschaftlerinnen und Rechtswissenschaftler beteiligten sich allerdings kaum an der Debatte, obwohl - oder gerade weil - die juristischen Verlage in der Schweiz gegenüber Open Access eine kritische Haltung einnahmen. ${ }^{6}$ Sie

* Dr. phil. Daniel Krämer ist wissenschaftlicher Mitarbeiter beim Schweizerischen Nationalfonds zur Förderung der wissenschaftlichen Forschung.

1 M. Hagner, Gute Bücher benötigen Zeit und Papier, in: NZZ, 23. Mai 2014; N. Bauermeister, Les sciences humaines en voie de précarisation, in: Le Temps, 17. Oktober 2014.

2 U. J. Wenzel, Open Access. Der Nationalfonds kapituliert, in: NZZ, 27. Mai 2014; St. Stöcklin/M. Fuchs, Das gedruckte Buch ist unser Labor, in: UZH News, 17. September 2014.

3 M. Dommann, Empörung alleine reicht nicht. Streit um den Open Access, in: NZZ, 17. Juni 2014 ; C. Hirschi, Der Schweizerische Nationalfonds und seine Open-Access-Strategie. Das geisteswissenschaftliche Buch im digitalen Zeitalter, in: NZZ, 19. Mai 2014; Stöcklin/Fuchs, Das gedruckte Buch; J. Adema, The Monograph Crisis Revisited, in: Open Reflections, 29. Januar 2015.

4 B. Hirschmann/D. Verdicchio, Open Access in der Schweiz, in: K. Söllner/B. Mittermaier (Hrsg.), Praxishandbuch Open Access, Berlin 2017, S. 215-222, hier S. 215.

5 Fonds national suisse de la recherche scientifique FNS-SNF: L'édition académique en danger! Die akademischen Verlage sind in Gefahr! URL: https://secure.avaaz.org/fr/community_petitions/Fonds_natio nal_suisse_de_la_recherche_scientifique_FNSSNF_Ledition_academique_en_danger_Die_akademische n_Verlage_sind_in/ (Stand vom 11.1.2019). Im Gegensatz zu Dike, Helbing Lichtenhahn, Orell Füssli, Schulthess und Stämpfli unterzeichnete der Schweizer Buchhändler- und Verlegerverband die Petition nicht.

6 D. Hürlimann/A. Grossmann, Open Access als Utopie?, in: Informationspraxis, 1/2017, S. 2-3. 
verwiesen oft auf die im deutschen Sprachraum für die Rechtswissenschaften typische Verflechtung zwischen Forschung und Praxis und den besonderen Charakter von Publikationen wie Kommentaren und Lehrbüchern, die häufig mehrmals aufgelegt würden und anderen Marktmechanismen gehorchten. ${ }^{7}$ Die Open-AccessStrategie des Bundes bringe die kleinen Wissenschaftsverlage in "existentielle Gefahr». ${ }^{8}$

Die Besorgnis der wissenschaftlichen Verlage und ihrer Autorinnen und Autoren schlug sich auch auf der politischen Ebene nieder. Nach einer Interpellation mit dem Titel «Open Access. Eine Bedrohung für das Verlagswesen?» beschäftigen sich im Frühjahr 2014 der Schweizerische Ständerat und der Schweizerische Bundesrat mit der Thematik. Obwohl der Bundesrat die Politik des SNF stützte, wünschten sich in der Diskussion der Interpellation die Ständerätinnen und Ständeräte einen Dialog zwischen den wissenschaftlichen Verlagen und dem SNF über seine OpenAccess-Politik. ${ }^{9}$

Was hatte die Differenzen ausgelöst? Die Schweizerische Akademie für Geistesund Sozialwissenschaften (SAGW) und der SNF unterzeichneten die Berliner Erklärung im Januar 2006. ${ }^{10}$ Gemeinsam suchten sie ab 2009 das Gespräch mit den wissenschaftlichen Verlagen zu Open Access bei Monographien. ${ }^{11} \mathrm{Im}$ Frühjahr 2014 passte der SNF schliesslich seine Publikationsförderung an und richtete sie auf die digitale Verbreitung aus: Statt wie bisher Monographien subsidiär zu fördern - es war stets ein Selbstbehalt von mindestens 3000 Franken vorgesehen -, unterstützte er die Druckvorstufe und die digitale Aufbereitung mit Pauschalen und er verlangte eine Open-Access-Veröffentlichung nach einer Frist von maximal 24 Monaten nach dem Erscheinungsdatum der gedruckten Version. Gleichzeitig wurde die Beantragung vereinfacht, indem Fördermittel für Monographien in das Budget von Gesuchen integriert werden konnten (vgl. dazu auch Tabelle 1). ${ }^{12}$

7 Für ein aktuelles Beispiel siehe die Paneldiskussion «Die Perspektive der Verlage» an der Tagung Open Access für die Rechtswissenschaft Pflicht oder Privatsache? am 19. Oktober 2018 in Frankfurt a.M. Der SNF fördert keine Lehrbücher. Zu Kommentaren siehe auch: A. Severin/M. Egger/M. P. Eve/D. Hürlimann, Discipline-Specific Open Access Publishing Practices and Barriers to Change, in: 1000Research 7 (11. Dezember 2018): 1925, hier S. 17.

8 D. Landolf, Sesam, öffne Dich, in: Schweizer Buchhandel, 4/2017, S. 11-13, hier S. 13.

9 Schweizer Parlament, 14.3215 Interpellation. Open Access. Eine Bedrohung für das Verlagswesen?, 20.3.2014, URL: https://www.parlament.ch/de/ratsbetrieb/suche-curia-vista/geschaeft?AffairId=201 43215. Sowie: Amtliches Bulletin, Ständerat, Sommersession 2014, Neunte Sitzung, 16.06.14, 14.3215, URL: https://www.parlament.ch/de/ratsbetrieb/amtliches-bulletin/amtliches-bulletin-die-ve rhandlungen? SubjectId=27044 (Stand vom 11.1.2019).

10 SAGW-Bulletin, 4/2008, S. 45. Zur Berliner Erklärung siehe: https://openaccess.mpg.de/Berliner-Erkl aerung (Stand vom 11.1.2019).

11 Zum europäischen Stand der damaligen Diskussion siehe: European Commission (Hrsg.), Open Access. Opportunities and Challenges, Brüssel 2008.

12 Neue Publikationsförderung: Auf digitale Verbreitung und Open Access ausgerichtet, URL: http:// www.snf.ch/de/fokusForschung/newsroom/Seiten/news-140416-publikationsfoerderung-digitale-verbreitung-open-access.aspx (Stand vom 11.1.2019). 


\section{B. Das Pilotprojekt OAPEN-CH}

Um die Unstimmigkeiten zu beseitigen, lancierte der SNF zusammen mit sozialund geisteswissenschaftlichen Verlagen aus der Schweiz und Deutschland das Pilotprojekt OAPEN-CH. Das Design der Studie lehnte sich stark an ähnliche Projekte in den Niederlanden und in Grossbritannien an. ${ }^{13}$ Mit der Studie sollten auf der einen Seite das gegenseitige Verständnis gefördert und die Transition zu Open Access bei Monographien begleitet werden, auf der anderen Seite wurden Daten zu den Kosten, zur Sichtbarkeit, zur Nutzung, zur Reichweite und zu den Verkaufszahlen von frei zugänglichen und gedruckten Monographien gesammelt. Die Autorinnen und Autoren der Bücher im Pilotprojekt konnten sich in einer Umfrage zudem zu ihrer Wahrnehmung und ihren Erwartungen an Open-Access-Monographien äußern. ${ }^{14}$

Am Pilotprojekt beteiligten sich zwölf Verlage mit 105 Monographien, wobei 53 Bücher in einer Open-Access-Version erschienen. ${ }^{15}$ Mit Carl Grossmann partizipierte lediglich ein juristischer Verlag an der Studie. ${ }^{16} \mathrm{Im}$ Rahmen des Pilotprojekts wurden daher weder Kommentare noch Lehrbücher veröffentlicht, weshalb die juristischen Verlage die Resultate von OAPEN-CH für sich im Vergleich zu kleineren und mittelständischen Wissenschaftsverlagen in anderen Disziplinen in den Geistes- und Sozialwissenschaften als weniger repräsentativ erachten dürften. ${ }^{17}$

Die Resultate des Pilotprojekts deckten sich grossmehrheitlich sowohl mit den Ergebnissen der beiden Projekte in den Niederlanden und in Grossbritannien wie auch mit denjenigen anderer internationaler Studien. ${ }^{18}$ Bei der Interpretation der Befunde muss berücksichtigt werden, dass Open Access bei Monographien während der Projektlaufzeit noch in den Kinderschuhen steckte. Sobald die Transition

13 E. Ferwerda/R. Snijder/J. Adema, OAPEN-NL. A Project Exploring Open Access Monograph Publishing in the Netherlands. Final Report, Den Haag 2013; E. Collins/C. Milloy, OAPEN-UK Final Report: A Five-Year Study into Open Access Monograph Publishing in the Humanities and Social Sciences, London 2016. Zum Studiendesign von OAPEN-CH siehe Kap. 3 des Schlussberichts von OAPEN-CH: E. Ferwerda/R. Snijder/B. Arpagaus/R. Graf/D. Krämer/E. Moser, „OAPEN-CH Auswirkungen von Open Access auf wissenschaftliche Monographien in der Schweiz. Ein Projekt des Schweizerischen Nationalfonds, Bern 2018.

14 Ferwerda et al., OAPEN-CH, S. 11.

15 Chronos, Editions Alphil, Edizione Casagrande, Librairie Droz, Schwabe, Seismo, Peter Lang und transcript beteiligten sich an beiden Ausschreibungen mit 96 Monographien. Carl Grossmann, Editions IES, Librum und Wallstein nahmen an einer Ausschreibung teil und publizierten im Rahmen des Pilotprojekts neun Monographien. Vgl. dazu Anhang 3 des Schlussberichts von OAPEN-CH: Ferwerda et al., OAPEN-CH.

16 Obwohl Peter Lang in Deutschland in den Rechtswissenschaften zahlreiche Bücher verlegt, stammten die Monographien im Pilotprojekt ausschliesslich aus anderen sozial- und geisteswissenschaftlichen Disziplinen.

17 Gesuche zur Finanzierung von Kommentaren sind reglementarisch nicht ausgeschlossen, aber selten. $\mathrm{Zu}$ den eingeschlossenen Publikationsgattungen siehe Kap. 3 des Schlussberichts von OAPEN-CH: Ferwerda et al., OAPEN-CH.

18 Für eine Übersicht zur Literatur siehe Kap. 2.1.6 des Schlussberichts von OAPEN-CH: Ferwerda et al., OAPEN-CH. 
weiter fortgeschritten ist, dürften zum Beispiel die Bibliotheken die Prozesse für die Akquisition von Monographien anpassen, was sich auf die Verkaufszahlen auswirken könnte:

- Die Verkaufszahlen wurden durch Open Access weder positiv noch negativ beeinflusst. Die Zahl der verkauften gedruckten und digitalen Exemplare ging nach sechs Monaten sehr stark zurück: $\mathrm{Zu}$ diesem Zeitpunkt hatten die Verlage bereits 76 Prozent aller im Untersuchungszeitraum veräußerten Exemplare verkauft, nach 12 Monaten waren es 87 Prozent und nach 24 Monate sogar 95 Prozent.

- Open Access hatte einen positiven Einfluss auf die Auffindbarkeit und die Sichtbarkeit von Monographien. Insbesondere die Ablage von Büchern auf verschiedenen Plattformen erwies sich als vorteilhaft: OAPEN-CH legte deshalb nicht nur eine Ablage auf institutionellen Repositorien und in Bibliothekskatalogen nahe, sondern auch auf der Website des Verlags und auf internationalen Plattformen wie der OAPEN Library oder (kommerziellen Anbietern wie) Google Books.

- Die Ablage von Open-Access-Monographien auf der OAPEN Library erhöhte die internationale Reichweite. In 136 Ländern wurde mindestens ein Download registriert. Weil über 90 Prozent der Publikationen im Pilotprojekt auf Französisch und Deutsch erschienen, wurden die Bücher vor allem in Frankreich und in Deutschland genutzt. Interessanterweise wurden auch in Italien und in den Vereinigten Staaten mehr Downloads verzeichnet als in der Schweiz selbst.

- Open Access hatte bei den Pilotbüchern einen Einfluss auf die Nutzung von Monographien. Auf Google Books wurden Open-Access-Bücher häufiger genutzt als Publikationen, deren Inhalt nur eingeschränkt zugänglich war (Zahl der Klicks auf Monographien, Zahl der aufgerufenen Seiten, Zahl der Downloads). Angesichts der relativ kleinen Stichprobe und des relativ kurzen Untersuchungszeitraums traten bei der Analyse der Nutzung allerdings große Unterschiede auf.

- Die Kosten für eine digitale Monographie variierten im Pilotprojekt sehr stark. Sie reichten von gut 7'200 Franken bis zu rund 38'000 Franken. Die Unterschiede ließen sich nicht leicht erklären, dürften aber die unterschiedlichen Geschäftsmodelle, Arbeitsprozesse und Budgetierungspraxen der teilnehmenden Verlage widerspiegeln. Sie konnten die effektiven Kosten ihrer Leistungen bei der Herstellung einer Monographie abrechnen (zum Beispiel Lektorat, Korrektorat, Layout, Satz, Peer-Review-Prozess, Bildrechte, Marketing und Distribution). Die Druckkosten lagen durchschnittlich bei 4'368 Franken. ${ }^{19}$

19 Die Daten in den Aufzählungen finden sich in: E. Ferwerda/R. Snijder/B. Arpagaus/R. Graf/D. Krämer/E. Moser, OAPEN-CH - The Impact Of Open Access On Scientific Monographs In Switzerland. A Project Conducted By The Swiss National Science Foundation (SNSF), Bern 2018. 
- Eine Mehrheit der Autorinnen und Autoren verfügte noch über wenig Erfahrungen mit Open-Access-Publikationen. Gleichwohl stand sie Open Access positiv gegenüber. Sie erhoffte sich nicht nur eine raschere Verbreitung der Forschungsresultate und eine höhere Sichtbarkeit, sondern auch mehr Zitierungen. Ein zentraler Punkt war für die Autorinnen und Autoren der Pilotbücher die Qualitätssicherung durch die Verlage: Auf der einen Seite durch herkömmliche Verlagsleistungen wie Lektorat und grafische Aufbereitung, auf der anderen Seite durch ein Peer-Review-Verfahren zur Sicherung der inhaltlichen Qualität. Sie unterstützten auch die Ablage der Publikationen auf verschiedenen Repositorien, um die Dissemination zu erleichtern und die Sichtbarkeit zu erhöhen.

- Die Verlage verfolgten weiterhin ein duales Geschäftsmodell, das heißt, neben der Open-Access-Version erschien stets auch eine gedruckte Ausgabe der Monographie. Im Rahmen des Pilotprojekts bauten sie zudem ein Peer-Review-Verfahren auf und sie entwickelten Geschäftsmodelle, um die anfallenden Kosten decken zu können. Eine Book Processing Charge (BPC) erachteten sie als ehrlicheres Geschäftsmodell als den grünen Weg mit einer Embargoperiode von 24 Monaten. Bei den Creative-Commons-Lizenzen sprachen sie sich für restriktive Lizenzen aus, um die kommerzielle Weiternutzung und eine Zerstückelung der Monographien zu verhindern. ${ }^{20}$

Im Verlaufe des Pilotprojekts räumten die wissenschaftlichen Verlage in den Geistes- und Sozialwissenschaften und der SNF die wichtigsten Differenzen aus. Die anfängliche Skepsis der Verlage wich während des Pilotprojekts und mündete in einem Open-Access-Modell, welches den Verlagen weiterhin die Herstellung von hochwertigen Monographien ermöglicht. ${ }^{21}$ Weder wird auf Kosten der Geistesund Sozialwissenschaft gespart noch entsteht durch die vorgesehene Langzeitarchivierung mit einem vorgeschriebenen Set von Metadaten ein digitales Ghetto. Schließlich bietet das Modell auch genügend Flexibilität, um die verschiedenen $\mathrm{Pu}$ blikationskulturen zu berücksichtigen. Aus juristischer Perspektive steht Open Access bei staatlich geförderten Forschungsergebnissen zudem nichts im Wege. ${ }^{22}$

\section{Umfassende Förderung von Open Access}

Die Ergebnisse des Pilotprojekts flossen in die neue Open-Access-Politik des SNF ein, die im internationalen Vergleich progressiv ist und sich an die Bedürfnisse der Forschenden und der kleineren Wissenschaftsverlage in der Schweiz anlehnt. Sie wurde im April 2018 lanciert und ist in die nationale Open-Access-Strategie von

20 Siehe für wichtigsten Resultate der Pilotstudie Kap. 4-6: Ferwerda et al., OAPEN-CH.

21 Siehe dazu insbesondere S. 67 in: Ferwerda et al., OAPEN-CH.

22 R. M. Hilty/M. Seemann, Open Access - Zugang zu wissenschaftlichen Publikationen im schweizerischen Recht. Rechtsgutachten im Auftrag der Universität Zürich, Zürich 2009; A. Kley, Freie Bahn für Open Access, in: plädoyer 3/2018, S. 36-38; D. H. Haux/F. S. Graf, Obligation to Open Access, in: sui generis, 2018.; Severin et al. Discipline-Specific Open Access Publishing. 
2017 sowie in den Aktionsplan Open Access von 2018 eingebettet. ${ }^{23}$ Die Politik des SNF ist in einigen Punkten allerdings ambitionierter als die nationale Strategie: Einerseits strebt der SNF bis 2020 und nicht bis 2024 den freien Zugang zu allen Publikationen an, die mehrheitlich mit Mitteln der öffentlichen Hand finanziert worden sind. Andererseits akzeptiert er zwar Green Open Access, der Fokus seiner Politik liegt aber auf dem goldenen Weg. ${ }^{24}$ Sowohl swissuniversities wie auch der SNF verfolgen mit ihren Strategien das Ziel, die unterschiedlichen Publikationskulturen in den verschiedenen Disziplinen zu respektieren und die Transformation mit einem gezielten Einsatz der (finanziellen) Ressourcen zu erleichtern.

23 Die nationale Strategie wurde von swissuniversities in Zusammenarbeit mit dem SNF im Auftrag des Staatssekretariats für Bildung, Forschung und Innovation (SBFI) ausgearbeitet. Der Aktionsplan dient der Umsetzung der nationalen Strategie. Vgl. dazu: https://www.swissuniversities.ch/themen/ digitalisierung/open-access sowie https://www.swissuniversities.ch/fileadmin/swissuniversities/Dokumente/Hochschulpolitik/Open_Access/Plan_d_action-d.pdf (Stand vom 11.1.2019). Gemäß einer Finanzflussanalyse gaben die Bibliotheken der Schweizer Hochschulen 2015 insgesamt 70 Millionen Franken für Lizenzen und Abonnemente an Verlage aus. Siehe dazu: Cambridge Economic Policy Associates Ltd, „Financial Flows in Swiss Publishing“; A. Machado/L. Hoppmann, „Bibliometric Study of the Swiss Publication System, 2016“.

24 Der SNF unterstützt den europäischen Plan S, er unterzeichnete ihn bisher aber nicht, weil der grüne Weg in der nationalen Open-Access-Strategie enthalten ist. Vgl. dazu: https://oa100.snf.ch/de/newsde/freier-zugang-zu-publikationen-der-snf-unterstuetzt-den-europaeischen-plan-s/ (Stand vom 11.1.2019). 
Tab. 1: Entwicklung der Open-Access-Politik des SNF * Kosten gemäss des Open-Access-Angebots des Verlags.
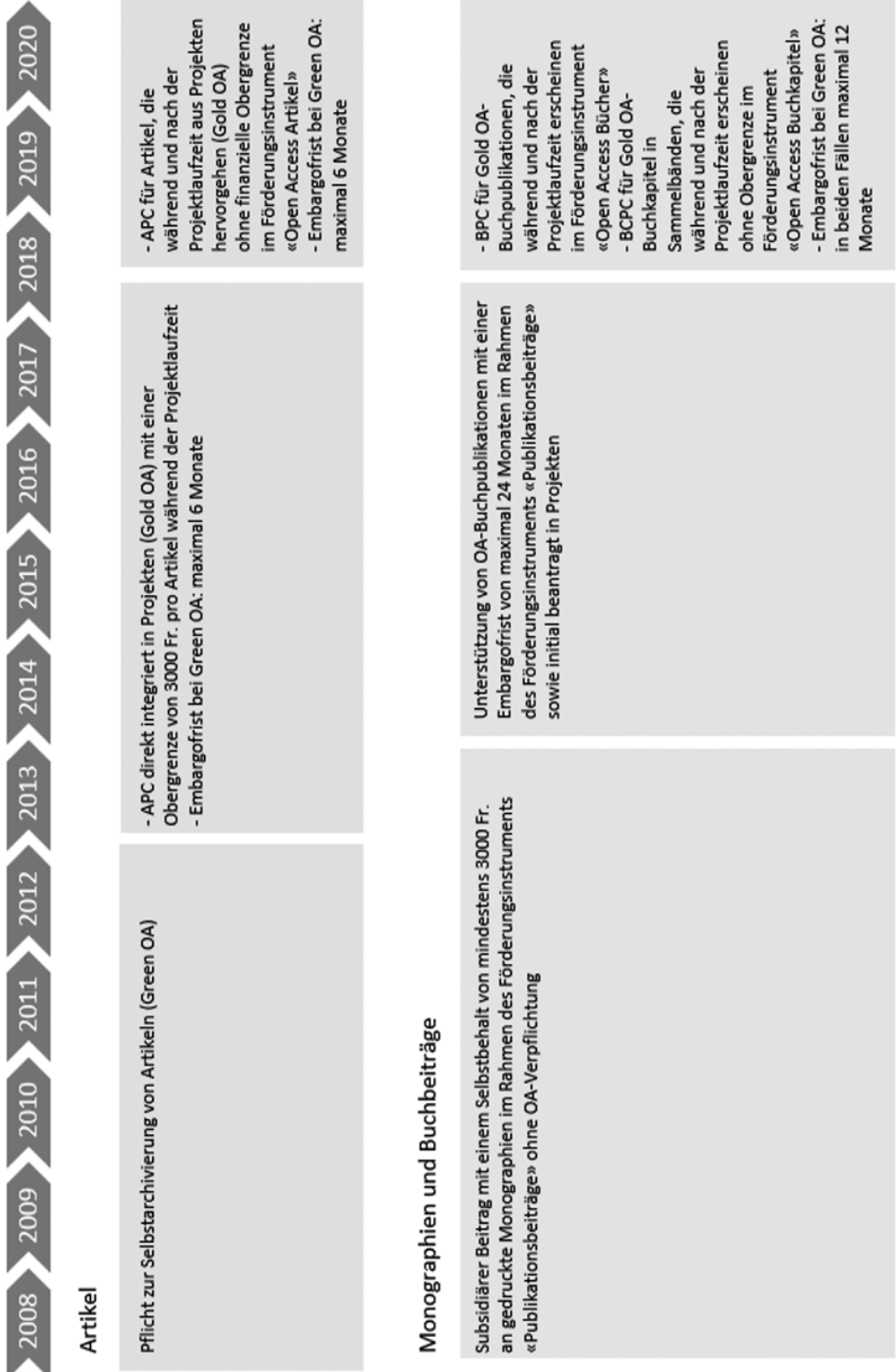
Bei der Förderung der Monographien entschied sich der SNF für ein modulares BPC-Modell. Neben den Resultaten aus dem Pilotprojekt stützte er sich bei der Entwicklung des Modells auf die langjährigen Erfahrungen der OAPEN Foundation, des österreichischen Fonds zur Förderung der wissenschaftlichen Forschung (FWF) und eine breit angelegte Studie zu Open-Access-Monographien in Europa. ${ }^{25}$ Im Basismodul werden die effektiv anfallenden Kosten bis zu 15'000 Franken für eine zitierfähige Open-Access-Version der Publikation abgegolten. Die Verlage können für eine Monographie bis 750'000 Zeichen unter anderem Leistungen zur Sicherung der Qualität (Peer-Review-Verfahren, Lektorat/Korrektorat, Satz, Layout, Cover und Bildbearbeitung), zur Bereitstellung der Metadaten (inkl. der Vergabe einer Creative-Commons-Lizenz und eines DOI) und der Veröffentlichung der Publikation auf ihrer Website geltend machen. In Zusatzmodulen sind schliesslich Mehrkosten durch eine höhere Zeichenzahl, einen höheren Aufwand für Layout und Bildrechte, zusätzliche Funktionen und digitale Anreicherungen sowie ein Fremdsprachenlektorat anrechenbar (siehe dazu Tab. 2). ${ }^{26}$

Tab. 2: Modulares BPC-System * Kosten gemäss des Open-Access-Angebots des Verlags.

\begin{tabular}{|l|l|}
\hline Modul & Höhe der Beiträge \\
\hline Basismodul* & $\begin{array}{l}\text { Bis zu 15'000 Fr. für eine zitierfähige OA-Publikati- } \\
\text { on bis 750'000 Zeichen }\end{array}$ \\
\hline Zusatzmodul Höhere Zeichenzahl & $\begin{array}{l}\text { Pauschal 3’000 Fr. für bis zu 250'000 zusätzliche } \\
\text { Zeichen } \\
\text { Pauschal 5’000 Fr. für mehr als insgesamt } \\
1 \text { Million Zeichen }\end{array}$ \\
\hline $\begin{array}{l}\text { Zusatzmodul Layout und Bildrech- } \\
\text { te** }\end{array}$ & Zusätzlich max. 5'000 Fr. \\
\hline Zusatzmodul Enriched E-Book*** & Zusätzlich max. 5'000 Fr. \\
\hline $\begin{array}{l}\text { Zusatzmodul Fremdsprachenlekto- } \\
\text { rat** }\end{array}$ & Zusätzlich max. 5'000 Fr. \\
\hline
\end{tabular}

* Kosten gemäss des Open-Access-Angebots des Verlags.

** Begründungspflichtiges Zusatzmodul, es muss eine Offerte beigelegt werden.

Die zweite wesentliche Neuerung bei den BPCs für Monographien (inkl. Dissertationen, Habilitationen und Kommentare) und Sammelbände war neben dem modularen System die Einführung der Qualitätssicherung durch die Verlage. Sie müssen für das publikationsreife, vollständige Manuskript mindestens ein Gutachten einholen. Anschliessend dokumentieren sie, wie allfällige Kritikpunkte bereinigt

25 Vgl. dazu: https://www.fwf.ac.at/de/forschungsfoerderung/open-access-policy/ (Stand vom 11.1.2019) sowie E. Ferwerda/F. Pinter/N. Stern, A Landscape Study On Open Access And Monographs, Bristol 2017.

26 Vgl. dazu: Reglement über die Open-Access-Publikationsförderung vom 7. November 2017, Art. 11. 
wurden und reichen die Gutachten zusammen mit der Dokumentation auf Aufforderung der Autorin oder des Autors ein. ${ }^{27}$

Eine dritte Neuerung im Bereich der Bücher war schliesslich die Einführung einer sogenannten Book Chapter Processing Charge (BCPC) für Beiträge in Sammelbänden, die auf dem goldenen Weg erscheinen. Mit Einschluss von Buchkapiteln sollen die Forschenden in den Geistes- und Sozialwissenschaften die Möglichkeit erhalten, eine häufig gewählte Form der Publikation von Forschungsresultaten sichtbarer zu machen und damit mehr Leserinnen und Leser zu erreichen. ${ }^{28}$

Zusammen mit dem FWF gehört der SNF in Europa zu einer der wenigen Förderorganisationen, die nicht nur Artikel, sondern auch Monographien in ihre OpenAccess-Politik eingeschlossen haben. ${ }^{29}$ Ähnliche Qualitätsstandards für die OpenAccess-Stellung von Büchern wie der FWF und der SNF entwickelte in Deutschland der Nationale Open-Access-Kontaktpunkt OA2020-DE in Zusammenarbeit mit Knowledge Unlatched und dem transcript Verlag. ${ }^{30}$ Vergleichbare Prinzipien und Empfehlungen enthält zudem ein Positionspapier zu Open-Access-Monographien von Science Europe, das im September 2019 publiziert worden ist. ${ }^{31}$

Bei der Lancierung der neuen Open-Access-Politik im Frühling 2018 passte der SNF auch die Förderung der Zeitschriftenartikel an. Er hob die Obergrenze von 3000 Franken für eine Article Processing Charge (APC) bis auf Weiteres auf und er fördert die Publikation von Artikeln in Gold-Open-Access-Zeitschriften auch nach dem Abschluss von Projekten, wenn der Artikel inhaltlich einen Bezug zum beendeten Projekt aufweist (vgl. dazu auch Tab. 1). ${ }^{32}$ Mit diesem Massnahmenpaket hofft der SNF, sein Ziel - 100 Prozent der aus seiner Förderung resultierenden $\mathrm{Pu}$ blikationen Open Access verfügbar zu machen - schon im Jahr 2020 zu erreichen.

\section{Erste Erfahrungswerte mit dem goldenen Weg für Bücher}

Eine Analyse der Entwicklungen seit dem Einbezug der Monographien in die Open-Access-Politik im Sommer 2014 lässt positive Rückschlüsse zu. Die Nachfra-

27 Bei Sammelbänden muss im Gutachten auf jeden Beitrag eingegangen werden, bei Dissertationen und Habilitationen können die Gutachten der Hochschule für die Qualifikationsschrift verwendet werden. Vgl. dazu: Reglement über die Open-Access-Publikationsförderung vom 7. November 2017, Art. 9.

28 In einer Übergangsphase bis Ende Dezember 2020 unterstützt der SNF auch Beiträge in Sammelbänden, die nicht auf dem goldenen Weg erscheinen. Vgl. dazu: Reglement über die Open-Access-Publikationsförderung vom 7. November 2017, Art. 19 Abs. 6.

29 Ferwerda/Pinter/Stern, Landscape Study; Severin et al., Discipline-Specific Open Access Publishing..

30 Vgl. dazu: https://oa2020-de.org/blog/2018/07/31/empfehlungen_qualitätsstandards_oabücher/ (Stand vom 11.1.2019).

31 Vgl. dazu: https://www.scienceeurope.org/our-resources/briefing-paper-on-open-access-to-academicbooks/ (Stand vom 26.9.2019).

32 Vgl. dazu insbesondere: Reglement über die Open-Access-Publikationsförderung vom 7. November 2017, Art. 3 Abs. 1a, Art. 7, Art. 10 und Art. 11 Abs. 1. 
ge nach Publikationsbeiträgen ging nicht zurück und der durchschnittlich bewilligte Betrag pro Monographie stieg um gut 25 Prozent an. Der SNF unterstützte die Publikation von Büchern in diesem Zeitraum mit mindestens 1,7 Millionen Franken pro Jahr. Allein für das Pilotprojekt OAPEN-CH wendete er zusätzlich 1,5 Millionen Franken auf. ${ }^{33}$

Die juristischen Verlage sind in der Schweiz mit Ausnahme von Carl Grossmann noch nicht auf den Open-Access-Zug aufgesprungen. Ähnlich wie bereits bei den Zeitschriftenartikeln nehmen sie eine abwartende Haltung ein, was wohl nicht nur der Verflechtung zwischen Forschung und Praxis in den Rechtswissenschaften geschuldet ist. $\mathrm{Zu}$ deren Charakteristika zählt auch die hohe Bedeutung nationaler Rechtssysteme, was zu zahlreichen Publikationen in den Landessprachen führt und die Wahl einheimischer Verlage begünstigt. Weil die Resultate aus der Forschung nicht nur für Akademikerinnen und Praktiker, sondern auch für Politikerinnen und Journalisten relevant sind, bereiten schweizerische Universitätsbibliotheken eine nationale Fachinformationsplattform für juristische Erst- und Zweitpublikationen vor. $^{34}$

Die geistes- und sozialwissenschaftlichen Verlage in der Schweiz entwickelten ihr Angebot für Open-Access-Monographien nach Abschluss des Pilotprojekts weiter und sie können den Transformationsprozess zu Open Access mit dem modularen BPC-Modell fortsetzen. In den ersten zehn Monaten seit der Einführung des BPCModells waren bereits 17 verschiedene Verlage an einem Gesuch beteiligt. Gleichzeitig wurde die neue Open-Access-Politik für Monographien auch von kleineren und mittelständischen Verlagen in Deutschland aufgenommen und grosse internationale Verlage haben ihr Angebot für den goldenen Weg ebenfalls ausgebaut. Die Publikationsfreiheit bleibt durch die neue Politik im Bereich der vom SNF geförderten Publikationsformen daher anders als befürchtet für die Gesuchstellerinnen und Gesuchsteller weitestgehend erhalten.

\section{E. Bibliographie}

Adema, Janneke. „The Monograph Crisis Revisited“. Open Reflections (blog), 29. Januar 2015. https://openreflections.wordpress.com/2015/01/29/the-monograph-crisisrevisited/.

Bauermeister, Nicole. „Les sciences humaines en voie de précarisation“. Le Temps, 17. Oktober 2014, 39716 Auflage.

Cambridge Economic Policy Associates Ltd. „Financial Flows in Swiss Publishing“. Zenodo, 12. Januar 2017. https://doi.org/10.5281/zenodo.240896.

33 Vgl. dazu die Datenbank P3 des SNF: http://p3.snf.ch/.

34 Severin et al., Discipline-Specific Open Access Publishing, S. 17; Aktionsplan, Anhang 3 (wie Anm. 23). 
Collins, Ellen, und Caren Milloy. „OAPEN-UK Final Report: A Five-Year Study into Open Access Monograph Publishing in the Humanities and Social Sciences“. Research Information Network, Januar 2016. http://oapen-uk.jiscebooks.org/files/2016/ 01/OAPEN-UK-final-report.pdf.

Dommann, Monika. „Empörung alleine reicht nicht. Streit um den Open Access“. NZZ. 17. Juni 2014.

European Commission. „Open Access. Opportunities and Challenges“. Brüssel, 2008. http://ec.europa.eu/research/science-society/document_library/pdf_06/open-access-ha ndbook_en.pdf.

Ferwerda, Eelco, Frances Pinter, und Niels Stern. „A Landscape Study on Open Access and Monographs: Policies, Funding and Publishing in Eight European Countries“. Zenodo, 1. August 2017. https://doi.org/10.5281/zenodo.815932.

Ferwerda, Eelco, Ronald Snijder, und Janneke Adema. „OAPEN-NL. A Project Exploring Open Access Monograph Publishing in the Netherlands. Final Report“. Den Haag: OAPEN Foundation, Oktober 2013.

Ferwerda, Eelco, Ronald Snijder, Brigitte Arpagaus, Regula Graf, Daniel Krämer, und Eva Moser. „OAPEN-CH - Auswirkungen von Open Access auf wissenschaftliche Monographien in der Schweiz. Ein Projekt des Schweizerischen Nationalfonds“. Zenodo, 23. April 2018. https://doi.org/10.5281/zenodo.1219172.

- „OAPEN-CH - The Impact Of Open Access On Scientific Monographs In Switzerland. A Project Conducted By The Swiss National Science Foundation (SNSF)“. Zenodo, 23. April 2018. https://doi.org/10.5281/zenodo.1216822.

Hagner, Michael. „Gute Bücher benötigen Zeit und Papier“. NZZ. 23. Mai 2014.

Haux, Dario Henri, und Fabienne Sarah Graf. „Obligation to Open Access: Academic Publishing of the Future? “ sui generis; sui generis, 2018. https://doi.org/10.21257/sg .63 .

Hilty, Reto M., und Matthias Seemann. „Open Access - Zugang zu wissenschaftlichen Publikationen im schweizerischen Recht. Rechtsgutachten im Auftrag der Universität Zürich“. Zürich, November 2009. https://www.hbz.uzh.ch/dam/jcr:a2d75ce8-26764efc-8f40-0f57644a3cff/OA_Rechtsgutachten_Hilty_de.pdf.

Hirschi, Caspar. „Der Schweizerische Nationalfonds und seine Open-Access-Strategie. Das geisteswissenschaftliche Buch im digitalen Zeitalter“. NZZ. 19. Mai 2014.

Hirschmann, Barbara, und Dirk Verdicchio. „Open Access in der Schweiz“. In Praxishandbuch Open Access, herausgegeben von Konstanze Söllner und Bernhard Mittermaier, 215-22. De Gruyter, 2017. https://www.degruyter.com/downloadpdf/books/ 9783110494068/9783110494068-025/9783110494068-025.xml

Hürlimann, Daniel, und Alexander Grossmann. „Open Access als Utopie?“ Informationspraxis Bd. 3 (16. Januar 2017): Nr. 1 (2017). https://doi.org/10.11588/ip.2017.1. 33687.

Kley, Andreas. „Freie Bahn für Open Access“. plädoyer 2018, Nr. 3 (28. Mai 2018): 36-38. 
Landolf, Dani. „Sesam, öffne Dich“. Schweizer Buchhandel, 4/2017.

Machado, Alexander, und Laura Hoppmann. „Bibliometric Study of the Swiss Publication System, 2016“. Zenodo, 18. November 2016. https://doi.org/10.5281/zenodo.1 67381.

Reglement über die Open-Access-Publikationsförderung vom 7. November 2017.

SAGW-Bulletin, 4/2008.

Severin, Anna, Matthias Egger, Martin Paul Eve, und Daniel Hürlimann. „DisciplineSpecific Open Access Publishing Practices and Barriers to Change: An Evidence-Based Review“. F1000Research 7 (11. Dezember 2018): 1925. https://doi.org/10.12688 /f1000research.17328.1.

Stöcklin, Stefan, und Marita Fuchs. „Das gedruckte Buch ist unser Labor“. UZH News, 17. September 2014.

Wenzel, Uwe Justus. „Open Access. Der Nationalfonds kapituliert“. NZZ, 27. Mai 2014. 